\title{
Diversités phénotypique et morphométrique des poulets locaux (Gallus gallus) de deux zones agroécologiques de Côte d'Ivoire*
}

\author{
Chia Valentine Yapi-Gnaore ${ }^{1}$ \\ N'goran Étienne Loukou' \\ Assanvo Simon Pierre N'Guetta ${ }^{2}$ \\ Boniface Kayang ${ }^{3}$ \\ Xavier Rognon ${ }^{4}$ \\ Michèle Tixier-Boichard ${ }^{5}$ \\ Gnénékita Touré ${ }^{1}$ \\ Yaya Coulibaly ${ }^{2}$ \\ Issaka Youssao ${ }^{6}$ \\ ${ }^{1}$ Centre national de recherche agronomique \\ Km 17 Route Dabou \\ 01 BP 1740 Abidjan \\ Côte d'Ivoire \\ <evayapi11@yahoo.fr> \\ $<$ nloukou@yahoo.fr> \\ <gnenekita@yahoo.fr> \\ ${ }^{2}$ Université de Cocody \\ UFR Biosciences \\ 22 BP 582
}

Abidjan 22

Abidjan

Côte d'Ivoire

<nguettaewatty@yahoo.fr>

<dcoulibaly_yahya@yahoo.fr>

${ }^{3}$ University of Legon

Department of Animal Science

PO Box LG 25

Legon

Ghana

<bbkayang@ug.edu.gh>

${ }^{4}$ Inra/INAP-G

UMR Génétique et diversité animale

78352 Jouy-en-Josas cedex

France

<xavier.rognon@agroparistech.fr>

${ }^{5}$ Inra-AgroParisTech

UMR1313 Génétique animale

et biologie integrative

Centre de recherches Inra

16, rue Claude Bernard

75231 Paris

France

<michele.tixier-boichard@recherche.gouv.fr>

${ }^{6}$ École polytechnique Abomé-Calavi

01 BP 209

Cotonou

Bénin

<issaka.youssao@epac.uac.bj>

Tirés à part : C.V. Yapi-Gnaore.

\section{Résumé}

Avec comme objectif une meilleure gestion des ressources génétiques avicoles, une caractérisation phénotypique et morphométrique de l'espèce Gallus gallus domesticus a été entreprise dans deux zones agroécologiques (forêt et savane) de Côte d'Ivoire. Un échantillon de 439 poules et 193 coqs a été photographié, décrit et mesuré individuellement. Une grande variabilité phénotypique dans la coloration et le dessin du plumage de ces poulets a été observée. Les colorations les plus fréquentes ont été le blanc $(11,0 \%$ en forêt ; $11,6 \%$ en savane) chez les coqs et poules, le rouge du corps avec une queue noire (9,3\% en forêt ; 6,7\% en savane) chez les coqs et la couleur perdrix ( $8,7 \%$ en forêt ; $6,4 \%$ en savane) chez les poules. Les poulets noirs étaient plus présents en zone de forêt $(9,8 \%)$ qu'en zone de savane $(3,3 \%)$ à l'inverse des poulets de couleur fauve ou rouge (5,5\% en forêt ; 9,2\% en savane). L'allèle E exprimant le noir étendu est fortement présent $(34,8 \%)$ dans cette population. Les mutations frisé, huppe et cou nu de fréquences respectives $2,4 \% ; 2,5 \%$ et $1,9 \%$ ont été observées. Un dimorphisme de poids vif (38,5 \%) a été observé entre les mâles et les femelles. Le poids vif a été fortement corrélé au périmètre thoracique avec un coefficient de 0,86 . Les populations de poulets des deux zones agroécologiques ne peuvent pas être différenciées. Différents gènes de coloration assurent une grande diversité de leur plumage qui joue un important rôle socioculturel.

Mots clés : Côte d'Ivoire ; phénotype ; morphométrie ; poulet.

Thèmes : amélioration génétique ; productions animales.

\section{Abstract \\ Phenotypic and morphometric diversities of local chicken (Gallus gallus) from two agroecological zones of Côte d'Ivoire}

With the goal of improving poultry genetic resource management, a phenotypic and morphometric characterization of Gallus gallus domesticus species was undertaken in two agroecologic zones (forest and savannah) of Côte d'Ivoire. A sample of 439 hens and 193 cocks were photographed, described and measured individually. Wide scale phenotypical variability was observed in colorings and patterns of the chickens' plumage. The most commonly observed colorings were white (11.0\% forest; $11.6 \%$ savannah) in cocks and hens, a red body with a black tail among cocks ( $9.3 \%$ forest $6.7 \%$ savannah) and a brown color of hens ( $8.7 \%$ forest ; 6.4\% savannah). Black chickens were more often present in the forest zone $(9.8 \%)$ than in the savannah zone $(3.3 \%)$ as opposed to the fawncolored or red coloured chickens (5.5\% forest ; $9.2 \%$ in savannah). The E allele expressing extended black is strongly present $(34.8 \%)$ in this population. The frizzled, crested and naked-neck mutations with respective frequencies of $2.4 \%, 2.5 \%$ and $1.9 \%$ were observed. Live weight dimorphism (38.5\%) was observed between males and females and was strongly correlated with the thoracic perimeter $(\mathrm{R}=0.86)$. The chicken populations of the two agroecological zones cannot be differentiated. However, various colouring genes ensure great diversity in their plumage, playing therefore an important socio-cultural role.

Key words: chickens; Ivory Coast; morphometry; phenotypes.

Subjects: animal productions; genetic improvement.

*Pour citer cet article: Yapi-Gnaore CV, Loukou NE, Kayang B, Rognon X, Tixier-Boichard M, Touré G, Coulibaly $Y$, N'Guetta ASP, Youssao Y. Diversités phénotypique et morphométrique des poulets locaux (Gallus gallus) de deux zones agroécologiques de Côte d'Ivoire. Cah Agric $2010 ; 19: 439-45$; doi : 10.1684/agr.2010.0436 
I aviculture est une source importante de revenu et d'apport en protéines animales (Bonfoh et al., 1997), participant ainsi significativement à la sécurité alimentaire (Guèye, 1998a). Dans les pays d'Afrique, les poulets traditionnels représentent environ $80 \%$ du total du cheptel avicole et contribuent pour une proportion non négligeable à la production de viande (25 à $70 \%$ ) et d'œufs (12 à $36 \%$ ) (Guèye, 1998b). Ces produits avicoles locaux se retrouvent au centre de nombreux événements de la vie sociale, culturelle et religieuse.

En Côte d'Ivoire, malgré le rôle socioéconomique important de la volaille locale, il n'existe pas de politique de gestion de l'aviculture traditionnelle. Les tentatives pour accroitre sa productivité par l'introduction de coqs améliorateurs (Zana et al., 1999), n'ont pas permis d'obtenir une amélioration significative des performances. La contribution du potentiel génétique des races locales à l'établissement de futures stratégies de gestion durable des ressources avicoles, nécessite une connaissance préalable des génotypes disponibles et de leur variabilité (Tixier-Boichard et al., 2009). Afin de mieux comprendre la diversité de cette volaille locale pour une meilleure utilisation des ressources avicoles, une caractérisation phénotypique et morphométrique des poulets traditionnels de Côte d'Ivoire a été réalisée.

\section{Matériel et méthode}

\section{Présentation des zones d'étude}

L'étude a été menée entre novembre 2006 et juillet 2007 dans deux zones agroécologiques (forêt et savane) de Côte d'Ivoire. Situé en Afrique de l'Ouest, le pays s'étend entre $4^{\circ} 30^{\prime}$ et $10^{\circ} 30^{\prime}$ de latitude Nord et entre $2^{\circ} 30^{\prime}$ et $8^{\circ} 30^{\prime}$ de longitude Ouest. La zone de forêt, située au sud-est et représentée par les circonscriptions administratives d'Aboisso, Alépé et Agboville, est caractérisée par un climat attiéen de pluviométrie moyenne annuelle $1600 \mathrm{~mm}$ et de température oscillant entre $26^{\circ} \mathrm{C}$ et $32{ }^{\circ} \mathrm{C}$. La zone de savane arborée située au centre-est couvrant Yamoussoukro, M'Bahiakro et Didiévi est caractérisée par un climat baouléen avec une pluviométrie

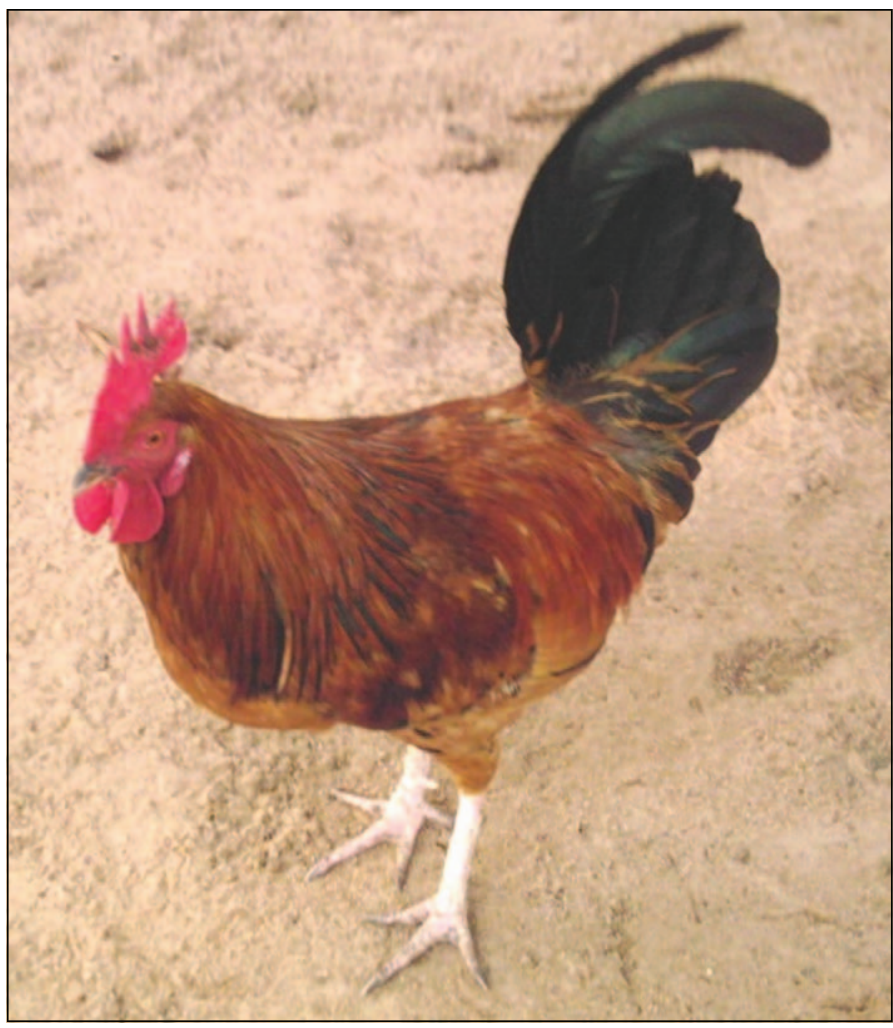

Figure 1. Coq rouge à queue noire présentant une crête simple de couleur rouge vif et aux tarses blancs.

Figure 1. Red cock with black tail showing simple red comb and white shanks.

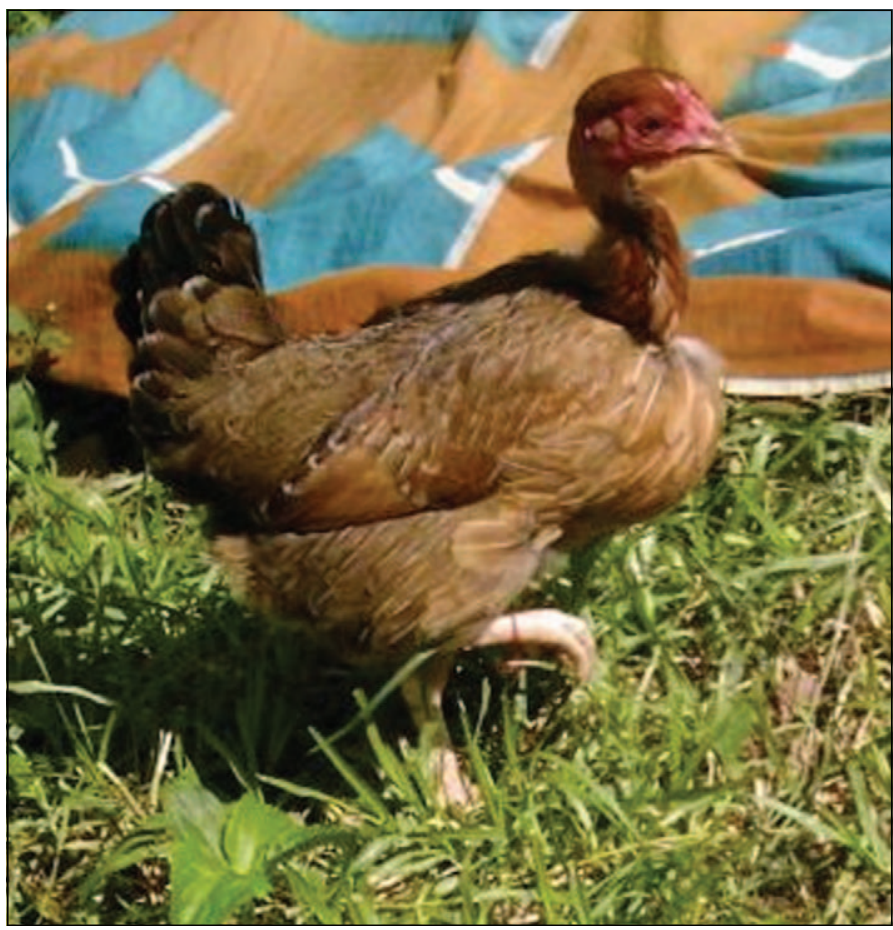

Figure 2. Poule au plumage " perdrix " doré à cou nu et à tarses blancs.

Figure 2. Naked neck brown coloured hen with white shanks. 
moyenne annuelle de $1000 \mathrm{~mm}$ et une température variant entre $22^{\circ} \mathrm{C}$ et $37{ }^{\circ} \mathrm{C}$ (ministère de l'Environnement et de la Forêt de Côte d'Ivoire, 1999).

\section{Matériel biologique et méthodes de collecte des données}

Un échantillon de 632 poulets ( 439 poules et 193 coqs), dont 131 utilisés pour la caractérisation morphométrique, a été constitué pour l'étude. Chaque poulet a été observé, photographié et décrit. Les données phénotypiques ont porté sur la couleur du plumage, de la peau, de la crête, des tarses, des oreillons et des yeux, la structure et la distribution du plumage, la forme de la crête et le squelette selon la nomenclature décrite par Coquerelle (2000) et les descripteurs de la FAO (1981). Les mesures des longueurs ont été prises à l'aide d'un mètre ruban gradué en millimètre et ceux des poids par une balance électronique de $5 \mathrm{~kg}$ de portée et de précision de 1 gramme.

\section{Analyses statistiques}

Les statistiques descriptives ont été obtenues à l'aide du logiciel SPSS version évaluation 16.1. Les fréquences alléliques ont été calculées selon Minvielle (1990) en considérant la population en équilibre panmictique. Une analyse factorielle des correspondances multiples (AFCM) a été réalisée sur 12 variables qualitatives avec le logiciel XLSTAT version 7.5. La procédure GLM du même logiciel a été utilisée pour analyser les variables quantitatives. Les coefficients de corrélation entre les différentes variables quantitatives et le poids vif ont été déterminés de même que la régression linéaire du poids sur le périmètre thoracique.

\section{Résultats}

\section{Caractérisation phénotypique}

Les poulets traditionnels ont présenté une grande variété de coloration du plumage. Six phénotypes de coloration ont une fréquence au moins égale à $5 \%$; il s'agit du blanc, du blanc sale, du noir, du fauve, du rouge à queue noire pour les coqs (figure 1), et de la couleur dite perdrix (figure 2) pour les poules portant l'allèle "brown " au locus E (tableau 1). Les poules à plumage froment (doré et argenté) sont faiblement observées. Les poulets noirs étaient plus présents en zone de forêt qu'en zone de savane, à l'inverse des poulets de couleur fauve plus fréquents en zone de savane. Le plumage de l'ensemble des poulets présentait quelques rares dessins. La barrure ou coucou (figure 3) a été la plus observée (tableau 1).

L'interprétation mendélienne des phénotypes permet d'identifier les allèles de coloration et de dessin du plumage les plus représentés dans la population. Il s'agit des allèles E, I, e $e^{b}, \mathrm{Co}, \mathrm{Db}$ et $\mathrm{S}$ avec des fréquences allant de 34,8 à 12,1 \% (tableau 2). L'allèle E responsable de la couleur noire étendue est majoritaire dans cette population; il est suivi par l'allèle I pour la couleur blanche et de l'allèle $\mathrm{e}^{\mathrm{b}}$ pour la coloration brune $\mathrm{du}$ type perdrix. Il n'existe pas de différence significative entre les fréquences alléliques d'une zone agroécologique à l'autre. Les mutations mo et $\mathrm{mo}^{\mathrm{p}}$ responsables respectivement des plumages cailloutés (2,4\%) et bariolés $(6,7 \%)$ étaient également présentes. Cependant les allèles $\mathrm{B}$

\section{Tableau 1. Fréquences phénotypiques (\%) des couleurs et des dessins du plumage.}

Table 1. Phenotypic frequencies (\%) of plumage colour and pattern.

\begin{tabular}{|c|c|c|c|}
\hline Phénotypes & Génotypes probables & Forêt & Savane \\
\hline Coloration chez les coqs et poules & & $n=328$ & $n=304$ \\
\hline Blanc & $($ ( I) ; (c c) & 11,0 & 11,6 \\
\hline Noir ${ }^{\pi}$ & E & 9,8 & 3,3 \\
\hline Gris $^{\pi}$ & (E BI) ; (E Lav) & 0,9 & 4,0 \\
\hline Rouge herminé & (s Co e) & 3,7 & 2,9 \\
\hline Blanc herminé & (S Co e) & 1,8 & 4,3 \\
\hline Fauve ou rouge & $\left(e^{b}\right.$ Co s) & 5,5 & 9,2 \\
\hline Blanc sale & $(\mid \mathrm{E}) ;((\mathrm{e} s) ; \lg (\mathrm{B} \mid \mathrm{BI})$ & 5,2 & 5,9 \\
\hline Coloration chez les coqs & & $n=108$ & $n=85$ \\
\hline Type sauvage doré ${ }^{\pi}$ & $\left(e^{+} s\right)$ & 2,1 & 4,1 \\
\hline Type sauvage argenté & $\left(e^{+} S\right)$ & 3,1 & 3,6 \\
\hline Rouge à queue noire & (Db s) & 9,3 & 6,7 \\
\hline Blanc à queue noire & (Db S) & 2,1 & 0,5 \\
\hline Bouleau & $\left(E^{R} S\right)$ & 1,0 & 1,0 \\
\hline Noir cuivré & $\left(E^{R} s\right)$ & 4,2 & 4,2 \\
\hline Coloration chez les poules & & $n=220$ & $n=219$ \\
\hline Type sauvage doré & $\left(e^{+} s\right)$ & 0,6 & 0 \\
\hline Noir poitrine maillée de brun & $\left(E^{R} s\right)$ & 2,7 & 3,2 \\
\hline Noir poitrine maillée d'argenté & $\left(E^{R} S\right)$ & 0,2 & 0,2 \\
\hline Froment doré & $\left(e^{w h} s\right)$ & 3,0 & 3,4 \\
\hline Froment argenté & $\left(e^{w h} S\right)$ & 0,7 & 1,1 \\
\hline Perdrix doré & $\left(e^{b} s\right)$ & 3,6 & 1,4 \\
\hline Perdrix argenté & $\left(e^{b} s\right)$ & 1,8 & 0,7 \\
\hline Perdrix claire doré & $\left(e^{b} s \mathrm{Db}\right)$ & 2,5 & 3,2 \\
\hline Perdrix claire argenté & $\left(e^{b} S\right.$ Db) & 0,7 & 1,1 \\
\hline Dessin chez les coqs et poules & & $n=328$ & $n=304$ \\
\hline Coucou argenté & $(B E S)$ & 3,2 & 1,7 \\
\hline Coucou doré & (B e s) & 2,5 & 1,1 \\
\hline Caillouté & (mo e) & 0,3 & 0,8 \\
\hline Noir caillouté & (mo E) & 1,7 & 1,9 \\
\hline Bariolé & $\left(\mathrm{mo}^{\mathrm{pi}} \mathrm{E}\right)$ & 4,0 & 3,3 \\
\hline
\end{tabular}

$\mathrm{n}=$ nombre d'oiseaux $;{ }^{\pi}$ : différence significative entre forêt et savane $(p<0,05)$. 


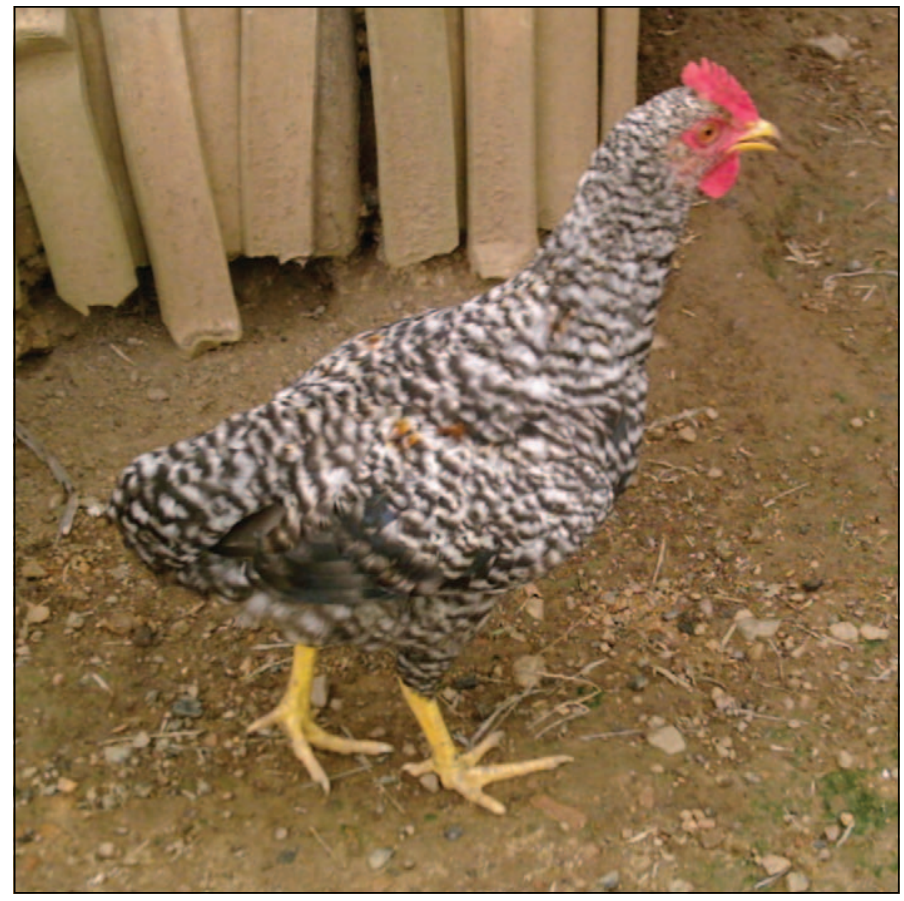

Figure 3. Coquelet argenté au plumage coucou présentant une crête simple de couleur rouge vif et aux tarses jaunes.

Figure 3. Silver barred cockerel with simple red comb and yellow shanks.

\section{Tableau 2. Fréquences (\%) de quelques allèles de coloration et dessin du plumage.}

Table 2. Some allele frequencies (\%) of plumage colour and pattern.

\begin{tabular}{lccc}
\hline Allèles & Ensemble & Forêt & Savane \\
\hline $\mathrm{E}$ & 34,8 & 37,5 & 32,0 \\
$\mathrm{I}$ & 28,1 & 27,1 & 29,0 \\
$\mathrm{e}^{\mathrm{b}}$ & 14,9 & 14,1 & 15,6 \\
$\mathrm{Co}^{\boldsymbol{\pi}}$ & 13,7 & $\mathbf{1 1 , 0}$ & $\mathbf{1 6 , 4}$ \\
$\mathrm{Db}^{\boldsymbol{\pi}}$ & 13,1 & $\mathbf{1 4 , 6}$ & $\mathbf{1 1 , 6}$ \\
$\mathrm{S}$ & 12,1 & 11,5 & 12,7 \\
$\mathrm{e}$ & 8,7 & 8,3 & 9,1 \\
$\mathrm{E}^{\mathrm{R}}$ & 8,4 & 8,2 & 8,6 \\
$\mathrm{e}^{+}$ & 6,8 & 5,8 & 7,8 \\
$\mathrm{Co}^{+}$ & 6,8 & 5,8 & 7,8 \\
$\mathrm{mo}^{\mathrm{pi}}$ & 6,7 & 4,9 & 8,6 \\
$\mathrm{~B}^{\boldsymbol{\pi}}$ & 4,3 & $\mathbf{5 , 7}$ & $\mathbf{2 , 9}$ \\
$\mathrm{B}^{\boldsymbol{\pi}}$ & 4,3 & $\mathbf{2 , 7}$ & $\mathbf{5 , 8}$ \\
$\mathrm{e}^{\text {wh }}$ & 4,1 & 3,6 & 4,6 \\
$\mathrm{mo}$ & 2,4 & 2,1 & 2,7 \\
\hline
\end{tabular}

$\pi$ : différence significative entre forêt et savane $(p<0,05)$.

(5.7\%) barré lié au sexe et $\mathrm{Db}(14,6 \%)$ noir restreint chez le mâle, étaient plus fortement présents en zone de forêt qu'en zone de savane où l'allèle $\mathrm{Bl}(5,8 \%)$ gris-bleu à l'état hétérozygote était le plus représenté.
Des mutations affectant la structure (F pour le plumage frisé) ou la répartition du plumage (Cr pour la huppe et Na pour le cou nu) sont observées avec une faible fréquence (tableau 3). La peau et les tarses jaunes, dus à la mutation $\mathrm{W}$, ont
Tableau 3. Distribution (\%) des phénotypes de structure, de coloration, des formes de plumes et de quelques parties du corps.

Table 3. Phenotype distribution (\%) for plumage structure, colour and feather and the format of some body parts.

\begin{tabular}{|c|c|c|}
\hline Phénotypes & Forêt & Savane \\
\hline \multicolumn{3}{|c|}{ Structure de la plume } \\
\hline Normale & 48,9 & 46,4 \\
\hline Frisée & 1,4 & 0,9 \\
\hline Autres & 1,6 & 0,8 \\
\hline \multicolumn{3}{|c|}{ Distribution du plumage } \\
\hline Normale & 48,8 & 46,1 \\
\hline Cou nu & 1,1 & 0,8 \\
\hline Huppe & 1,9 & 0,6 \\
\hline \multicolumn{3}{|c|}{ Couleur de la peau } \\
\hline Blanc $^{\pi}$ & 47,6 & 31,8 \\
\hline Jaune $^{\pi}$ & 4,3 & 16,3 \\
\hline \multicolumn{3}{|c|}{ Couleur des tarses } \\
\hline Blanc & 21,7 & 24,1 \\
\hline Jaune & 13,6 & 15,2 \\
\hline Noir & 14,7 & 7,8 \\
\hline Vert & 0,4 & 0,7 \\
\hline Autres & 1,6 & 1,4 \\
\hline \multicolumn{3}{|c|}{ Couleur des yeux } \\
\hline Orangé & 51,1 & 45,4 \\
\hline Autres & 0,8 & 2,7 \\
\hline \multicolumn{3}{|c|}{ Couleur des oreillons } \\
\hline Blanc & 37,7 & 38,3 \\
\hline Rouge & 13,1 & 8,6 \\
\hline Autres & 1,1 & 1,3 \\
\hline \multicolumn{3}{|c|}{ Couleur de la crête } \\
\hline Rouge vif & 45,9 & 41,6 \\
\hline Violacé & 2,9 & 4,4 \\
\hline Rouge pâle & 2,2 & 1,7 \\
\hline Autres & 1,0 & 0,3 \\
\hline \multicolumn{3}{|c|}{ Forme de la crête } \\
\hline Simple & 49,5 & 46,0 \\
\hline Rosacée & 1,4 & 1,6 \\
\hline Autres & 1,0 & 0,2 \\
\hline \multicolumn{3}{|c|}{ Squelette de I'oiseau } \\
\hline Normal & 50,6 & 46,7 \\
\hline Pattes courtes & 1,1 & 1,3 \\
\hline Autres & 0,2 & 0,2 \\
\hline
\end{tabular}

${ }^{\pi}$ : différence significative entre forêt et savane $(p<0,05)$. 
été observés à plus de $20 \%$, avec des différences non significatives, sauf pour la couleur de la peau.

L'AFCM indique que le plan F1-F2 décrit $21 \%$ de la variabilité totale. Les variables discriminantes sont la couleur du plu(DEPL), la couleur de crête (CCR), la couleur des tarses (CTA), la couleur des oreillons (COR) et la couleur de la peau (CPE). Les variables CTA et CCR contribuent le plus (11 \%) à l'axe F1. Sur l'axe F2, à $62 \%$, ce sont les variables CPL et DEPL. Le plan F1-F2, présentant les modalités des variables (figure 4) et les individus (figure 5) permet de définir trois groupes de poulets. Le groupe 1 (G1), du côté négatif de F1, est constitué des poulets à coloration diverse du plumage sans dessin (perdrix, blanc sale, rouge ou fauve), à tarses blancs, crête rouge vif ou violacée, oreillons blancs et épiderme blanc. Le groupe 2 (G2) se situant des côtés positif de F1 et négatif de F2 comporte des poulets à plumage froment (doré et argenté) avec des dessins barrure (coucou) et bariolé, à tarses et peau jaunes et oreillons rouges. mage (CPL), le dessin du plumage

Les oiseaux au plumage noir caillouté, possédant des tarses noirs, des oreillons rouges et une peau blanche, constituent le groupe 3 (G3) et se situent du côté positif de F1 et de F2.

\section{Caractérisation morphométrique}

Un dimorphisme du poids vif de $38,5 \%$ a été observé entre les mâles $(1,8 \mathrm{~kg})$ et les femelles $(1,3 \mathrm{~kg})$ des poulets. Les longueurs moyennes du corps et des tarses étaient supérieures chez le mâle $(23$ et $7,4 \mathrm{~cm}$ ) comparées à celles de la femelle $(20 \mathrm{~cm}$ et $5,8 \mathrm{~cm}$ ). Les poulets de la forêt ont une conformation plus grande que ceux de la savane, mais les différences ne sont pas significatives (tableau 4). Le phénotype " pattes courtes " traduisant la présence de la mutation nanisme a été rarement observé (tableau 3). Le poids vif a été positivement corrélé aux caractères longueur et pourtour du tarse, ainsi que longueur du corps; avec une corrélation forte $(\mathrm{R}=0,86)$ entre ce dernier et le périmètre thoracique. La régression linéaire du poids

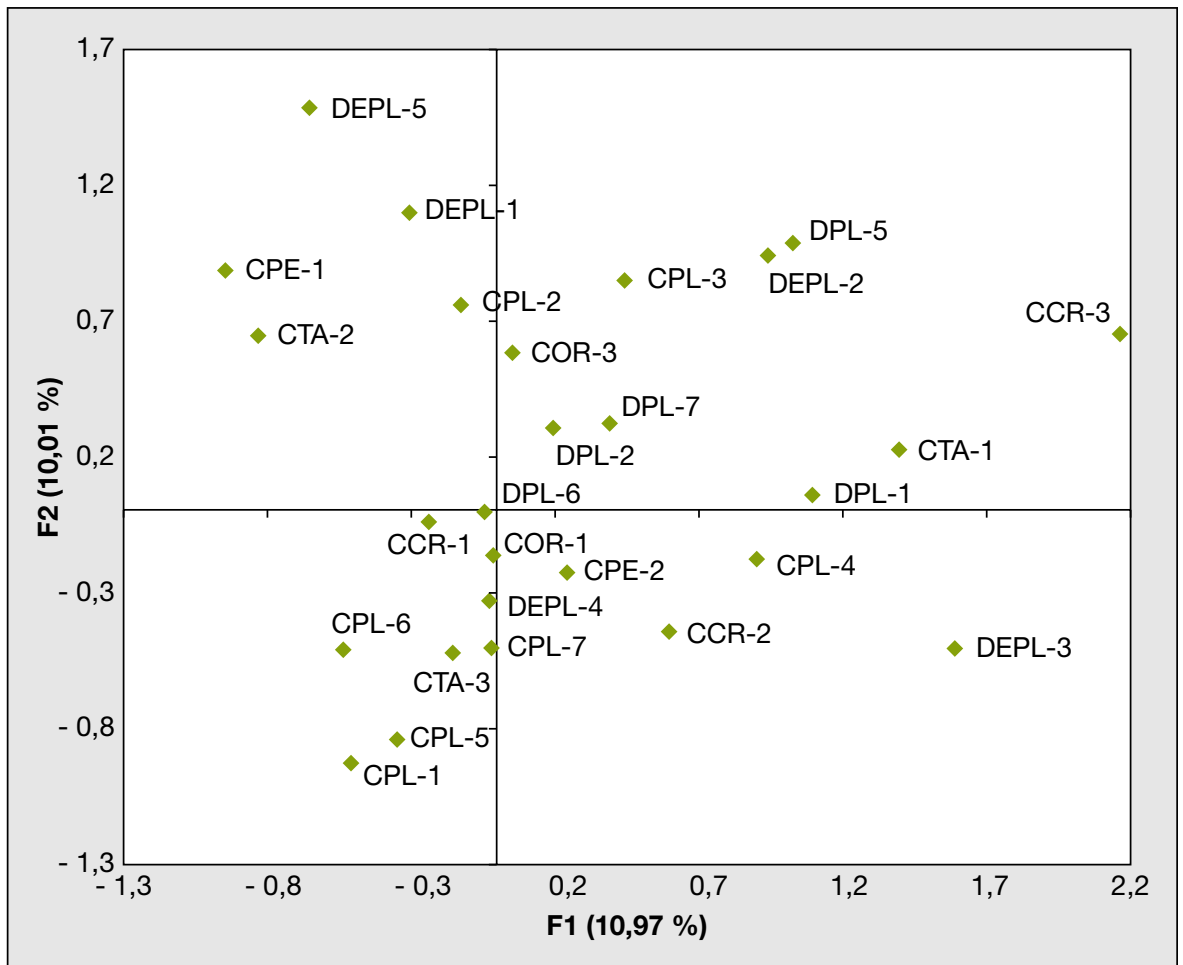

Figure 4. Représentation graphique des modalités des variables couleur plumage (CPL), dessin plumage (DEPL), couleur tarse (CTA), couleur oreillon (COR), couleur peau (CPE), couleur crête (CCR), distribution plumage (DPL) dans le plan F1-F2.

Figure 4. Graphic representation of the classes of variable feathering colours (CPL), feather patterns (DEPL), shank colour (CTA), earlobe colour (COR), skin colour (CPE), comb colour (CCR), feather structure (DPL) in the F1-F2 axes.

Le chiffre suivant la variable correspond à la modalité. vif (P) sur le périmètre thoracique (PT) a été définie par l'équation ci-dessous :

$$
\mathrm{P}=0,049 * \mathrm{PT}-0,022
$$

\section{Discussion}

Cette étude a montré une diversité de couleurs de plumage des poulets locaux de Côte d'Ivoire. Cette diversité n'est pas spécifique aux poulets traditionnels ivoiriens. Des observations similaires ont été faites par d'autres auteurs (Van Marle-Köster et Casey, 2001; Qu Lujiang et al., 2006 ; Fotsa, 2008) sur les poulets traditionnels. La plupart des gènes observés chez les poulets ivoiriens s'expriment sous plusieurs formes traduisant ainsi leur polymorphisme. Les fréquences phénotypiques obtenues diffèrent très peu d'une zone à l'autre, ce qui suppose que l'environnement influence peu l'expression de ces gènes. La présence de poulets au plumage noir plus importante dans la zone de forêt que dans la zone de savane, pourrait s'expliquer par la sélection naturelle en zone de forêt où le plumage noir, moins visible, serait davantage épargné par les prédateurs. L'allèle $\mathrm{E}$ responsable de cette coloration noire est très fréquent $(34,7 \%)$ dans la population des poulets ivoiriens, ce qui rejoint les résultats de Fotsa (2008) dans la zone forestière humide du Cameroun. L'allèle $\mathrm{e}^{\mathrm{b}}$ responsable de la coloration type perdrix ou fauve herminé, une mutation au locus $\mathrm{E}$, très présent $(14,9 \%)$ chez les poulets de Côte d'Ivoire, est certainement dû aux choix des éleveurs d'utiliser des reproductrices du type perdrix. Ce type de coloration est majoritaire $(37,3 \%)$ parmi les poules au Congo Brazzaville (Akouango et al., 2004) et au Cameroun (Fotsa, 2008), indiquant ainsi une probable origine commune des poulets traditionnels ou une préférence commune de la part des éleveurs de ces trois pays.

La typologie répartissant les poulets en trois groupes a été constituée à partir de poulets issus des deux zones, indépendamment du sexe et de la provenance. Les groupes ethniques de ces zones (Baoulé en zone de savane; Abey, Akyé et Agni en zone de forêt), appartiennent au grand groupe ethnique Akan, originaire du Ghana (Ekanza, 2007). Ces ethnies partageant presque les mêmes rituels (naissance et baptême d'enfants, cérémonies funèbres, accueil de visiteurs, apaiser la colère ou demander l'aide des ancêtres, 


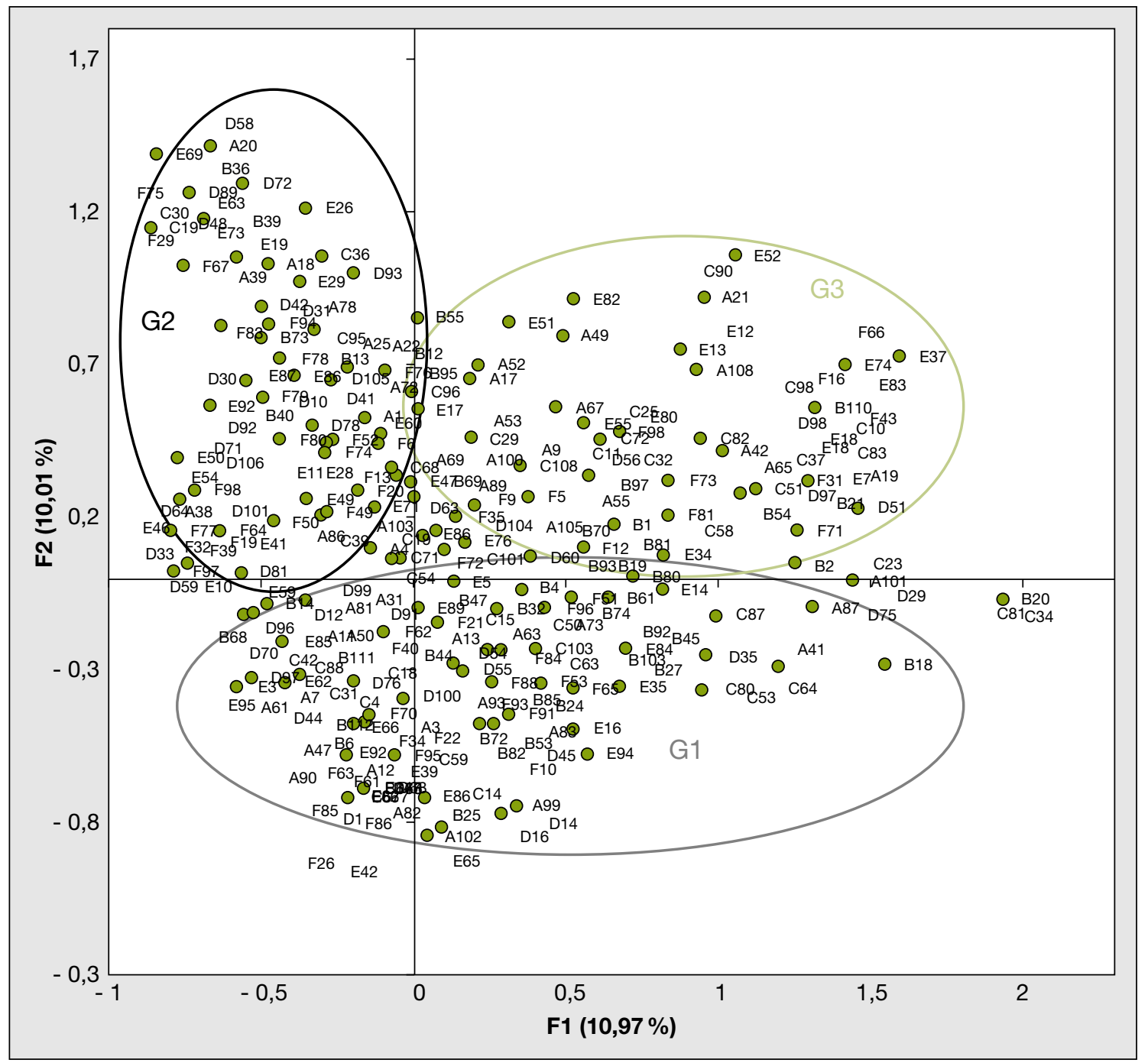

Figure 5. Représentation des groupes $\mathrm{G} 1, \mathrm{G} 2, \mathrm{G} 3$ des poulets de forêt (A, B, C) et de savane (D, E, F) dans le plan F1-F2.

Figure 5. Representation of groups G1, G2, G3 of forest (A, B, C) and savannah (D, E, F) chickens in the F1-F2 axes. Le chiffre indique le numéro d'identification.

\section{Tableau 4. Moyennes des caractères zootechniques des poulets traditionnels et leurs coefficients de corrélation par rapport au poids vif.}

Table 4. Mean values of zootechnical characteristics of local chickens and their correlation coefficients with body weight.

\begin{tabular}{lrrrrc}
\hline & Mâles & Femelles & Forêt & Savane & Corrélation/poids \\
\hline Poids vif $(\mathrm{kg})$ & $1,8 \pm 0,4^{\mathrm{a}}$ & $1,3 \pm 0,3^{\mathrm{b}}$ & $1,5 \pm 0,4^{\mathrm{a}}$ & $1,4 \pm 0,3^{\mathrm{b}}$ & - \\
Longueur tarse $(\mathrm{cm})$ & $7,3 \pm 0,8^{\mathrm{a}}$ & $5,8 \pm 0,6^{\mathrm{b}}$ & $6,3 \pm 0,9^{\mathrm{a}}$ & $5,9 \pm 0,8^{\mathrm{b}}$ & 0,70 \\
Longueur corps $(\mathrm{cm})$ & $23,4 \pm 1,4^{\mathrm{a}}$ & $20,4 \pm 2,4^{\mathrm{b}}$ & $21,3 \pm 2,4^{\mathrm{a}}$ & $20,7 \pm 2,6^{\mathrm{a}}$ & 0,59 \\
Longueur pilon $(\mathrm{cm})$ & $14,4 \pm 1,2^{\mathrm{a}}$ & $12,3 \pm 1,5^{\mathrm{b}}$ & $12,4 \pm 1,3^{\mathrm{a}}$ & $13,0 \pm 1,9^{\mathrm{b}}$ & 0,46 \\
Pourtour tarse $(\mathrm{cm})$ & $4,7 \pm 0,6^{\mathrm{a}}$ & $3,6 \pm 0,4^{\mathrm{b}}$ & $3,9 \pm 0,8^{\mathrm{a}}$ & $3,7 \pm 0,5^{\mathrm{a}}$ & 0,75 \\
Périmètre thoracique (cm) & $32,5 \pm 2,6^{\mathrm{a}}$ & $28,7 \pm 2,7^{\mathrm{b}}$ & $30,4 \pm 3,6^{\mathrm{a}}$ & $28,8 \pm 2,4^{\mathrm{b}}$ & 0,86 \\
Longueur crête $(\mathrm{cm})$ & $8,9 \pm 1,5$ & - & $9,2 \pm 1,6^{\mathrm{a}}$ & $8,6 \pm 1,5^{\mathrm{a}}$ & - \\
Longueur barbillon $(\mathrm{cm})$ & $4,5 \pm 1,0$ & - & $5,2 \pm 0,8^{\mathrm{a}}$ & $3,9 \pm 0,8^{\mathrm{b}}$ & - \\
\hline
\end{tabular}


etc.) ont adopté les mêmes critères de choix des géniteurs dont la couleur et la structure du plumage correspondent à ces exigences. La similarité des phénotypes peut être aussi due à un brassage de poulets favorisé par la facilité du transport et la mobilité des peuples avec leur volaille. La structure et la distribution des plumes, généralement normales, ont connu quelques mutations (F, Na, Cr) qui pourraient être exploitées. Le gène Na confère au poulet cou nu une meilleure tolérance à la chaleur ; caractéristique très importante pour les pays tropicaux comme la Côte d'Ivoire, soumis à de fortes températures. En conditions chaudes, ce type génétique est meilleur en production de viande et d'œufs que le type normal na (Coquerelle, 2000).

La plupart des poulets aux tarses jaunes ont la peau jaune, due à la présence de la mutation W qui entraîne un dépôt de pigments xanthophylles dans l'épiderme de la peau et des tarses (Mc Gibbon, 1974). Cette coloration des tarses et de la peau est celle des poulets du groupe G2, dont les poules sont essentiellement de plumage froment $\left(\mathrm{e}^{\mathrm{wh}}\right)$. L'allèle $\mathrm{W}$ proviendrait des lignées commerciales introduites dans les élevages traditionnels (Fotsa, 2008). De même, l'allèle $e^{\text {wh }}$, récessif par rapport aux allèles $\mathrm{E}$ et $\mathrm{E}^{\mathrm{R}}$ (plumage noir étendu ou noir restreint) est présent chez des races européennes (Coquerelle, 2000). Le dessin le plus observé, la barrure $\mathrm{B}$ liée au sexe, intervient dans le sexage des poussins d'un jour (Coquerelle, 2000). Sa présence dans la population locale pourrait être expliquée par l'introduction dans les élevages traditionnels de coqs améliorateurs au plumage coucou (Zana et al., 1999). Cette introduction a été certainement plus forte en forêt où une alimentation disponible plus abondante la rendait possible. Cela expliquerait la meilleure conformation des oiseaux de cette zone. Le bariolé ( $\mathrm{mo}^{\mathrm{pi}}$ ) et le caillouté (mo) sont des mutations au locus $\mathrm{Mo}^{+}$qui diminuent l'extension du noir sur tout le plumage (Coquerelle, 2000). Ces mutations contribuent fortement à la diversité des plumages de ces volailles dans le milieu rural. Cette diversité visible est cohérente avec la diversité moléculaire mise en évidence à l'aide de marqueurs microsatellites, montrant une grande variabilité génétique intrapopulation caractérisée par un taux élevé d'hétérozygotie et d'allèles efficaces (Loukou et al., 2009).

La morphométrie des poulets traditionnels est caractérisée par le dimorphisme de poids vif observé entre les coqs et les poules. Fotsa (2008) a montré que ce dimorphisme varie en fonction du caractère mesuré. Cependant, le dimorphisme lié au poids ou à la longueur de tarses est le plus marqué. Le dimorphisme de $38,5 \%$ pour le poids observé dans cette étude est un peu plus élevé que les valeurs estimées de 15,0 à 30,2\% sur la volaille locale du Cameroun (Fotsa, 2008). En milieu villageois, l'équation de prédiction du poids vif à partir du périmètre thoracique constitue une méthode simple pour la mesure du poids. Une étude similaire a été déjà menée par Guèye et al. (1998) qui ont obtenu une corrélation de 0,74 entre le poids vif et la circonférence du thorax.

\section{Conclusion}

Il ressort de cette étude qu'il n'est pas possible de différencier les poulets issus des deux zones agroécologiques (forêt et savane) de Côte d'Ivoire par leurs caractères phénotypique et morphométrique. Cependant, les gènes qui assurent la diversité de leur plumage ont été identifiés et sont présents chez les poulets des deux zones. Ces gènes confèrent à ces poulets différentes colorations et structures de plumage, sans doute en relation avec les exigences socioculturelles de leurs utilisations dans le milieu rural. La répartition en trois groupes des poulets traditionnels ivoiriens s'est faite à partir de six variables les plus discriminantes (couleurs du plumage, des tarses, des oreillons, de la peau, de la crête et dessins du plumage) quelle que soit la zone géographique. Le dimorphisme marqué entre les deux sexes peut permettre aux éleveurs, dans les conditions actuelles d'élevage en liberté, de sélectionner précocement les mâles pour la production de viande. La grande diversité phénotypique et la variabilité génétique observées peuvent être mises à profit (croisement pour améliorer la reproduction, sélection des mâles pour améliorer le poids, protection contre la prédation par camouflage et adaptation au milieu local) dans la valorisation et la stratégie de gestion durable des ressources avicoles locales.

\section{Remerciements}

Ce travail a été réalisé avec le soutien financier du projet DURAS (Promotion du Développement Durable dans les Systèmes de Recherche Agricole Sud) DCG-08.

\section{Références}

Akouango F, Mouangou F, Ganongo G. Phénotypes et performances d'élevage chez des populations locales de volailles du genre Gallus gallus au Congo Brazzaville. Cah Agric 2004; 13: 257-62.

Bonfoh B, Ankers P, Pfister K, Pangui LJ, Toguebaye BS. Répertoire de quelques contraintes de I'aviculture villageoise en Gambie et propositions de solutions pour son amélioration. Proceedings INFPD Workshop, M'Bour, Senegal, Dec. 9-13, 1997. 2000. www.fao.org/ag/AGAlnfo/ themes/es/infpd/documents/anrdp/mb27.rtf

Coquerelle G. Les poules: Diversité génétique visible. Paris : Inra éditions, 2000.

Ekanza SP. Origines, migration, peuplement. Le cas des populations ivoiriennes. Quelle méthodologie? 2007. www.histoire-afrique.org/ article165.html

FAO. Descripteurs des espèces avicoles. Banque de données des ressources génétiques animales. Rome: FAO, 1981.

Fotsa JC. Caractérisation des populations de poules locales (Gallus gallus) au Cameroun. Thèse de doctorat en génétique animale et systèmes de productions, Inra/AgroParistech, Paris (France), 2008AGPT0094, 2008. http://pastel. paristech.org/4904/01/THESE_FOTSA_B18_DEC_2008D_Agro_Paris_Tech.pdf

Guèye EF. Poultry plays an important role in African village life. World Poultry 1998a; 14 : 14-7.

Guèye EF. Village egg and fowl meat production in Africa. World Poultry Sci J 1998b ; 54 : 73-86.

Guèye EF, Ndiaye A, Branckaert RDS. Prediction of body weight on the basis of body measurements in mature indigenous chickens in Senegal. Livest Res Rural Dev 1998; 10 : article 103 www.cipav.org.co//rrd//rrd10/3/sene103.htm

Loukou NE, Yapi-Gnaoré CV, Touré G, et al. Evaluation de la diversité des poulets traditionnels de deux zones agroécologiques de Côte d'lvoire à l'aide de marqueurs microsatellites. J Anim Plant Sci 2009 ; 5 : 425-36.

Mc Gibbon WH. A shank color mutation in Cornell randombred SCW Leghorns. Poultry Sci $1974 ; 53: 1251-3$.

Minvielle F. Principes d'amélioration génétique des animaux domestiques. Paris: Inra editions, 1990.

Lujiang Q, Li X, Xu G, et al. Evaluation of genetic diversity in Chinese indigenous chicken breeds using microsatellite markers. Sci China Ser C 2006 ; $49: 305-8$.

Ministère de l'Environnement et de la Forêt de Côte d'Ivoire. Diversité Biologique de la Côte $d^{\prime} l v o i r e$. Rapport de synthèse. Abidjan (Côte d'Ivoire) : ministère de I'Environnement et de la Forêt, 1999. http://bch-cbd.naturalsciences.be/ civoire/contribution/monographie/monographie. pdf

Tixier-Boichard M, Bordas A, Rognon X. Characterisation and monitotring of poultry genetic resources. World Poultry Sci J 2009; 65: 272-85.

Van Marle-Köster E, Casey NH. Phenotypic characterisation of native chicken lines in South Africa. Anim Genet Resour Inf 2001; 29 : 71-8.

Zana O, Aman N, Kouassi NF, Zoumana C. Enquête sur la production avicole traditionnelle en milieu rural au nord de la Côte d'lvoire: cas de la zone dense de Korhogo. Agron Afr 1999; $11: 49-56$. 